\title{
HECKE THEORY OF SERIES FORMED WITH MODULAR SYMBOLS AND RELATIONS AMONG CONVOLUTION $L$-FUNCTIONS
}

\author{
NIKOLAOS DIAMANTIS \\ Max-Planck-Institute für Mathematik \\ CORMAC O'SULLIVAN \\ University of Maryland \\ E-mail: diamant@mpim-bonn.mpg.de \\ Fax number: (49)(228) 402277
}

\section{Introduction.}

In [G1], [G2], the distribution of modular symbols is studied and a new class of functions which satisfy a transformation law involving these objects is introduced. The goal of Goldfeld's program is to prove Szpiro's conjecture which states that for elliptic curves with minimal discriminant $D$ and conductor $N$ there is an absolute constant $\kappa$ such that $D \ll N^{\kappa}$. To do this, an equivalent conjecture involving modular symbols is established in [G4] using the, now proven, conjecture of Shimura, Taniyama and Weil. It seems that a sufficiently good understanding of the new series proposed by Goldfeld should yield a resolution of these conjectures. We repeat here their definition in a somewhat more general form to include period polynomials rather than modular symbols only.

For positive integers $M, N$ such that $M \mid N$, let $m, k$ be non-negative integers such that $m \geq k-2 \geq 0$ and let $\chi$ be a Dirichlet character modulo $N$. First, for each $f \in S_{k}(M)=\left\{\right.$ holomorphic cusp forms of weight $k$ and level $M$ \} we denote by $r_{f}$ the map which sends $\gamma \in \Gamma_{0}(M)$, the Hecke congruence group of level $M$, to the polynomial function:

$$
r_{f}(\gamma)(z):=\int_{i \infty}^{\gamma^{-1} i \infty} f(w)(w-z)^{k-2} d w .
$$

We then consider the functions $G^{*}$ on the upper-half plane $\mathfrak{H}$ of polynomial growth at the cusps such that for $f \in S_{k}(M)$ and $G \in M_{m}(N, \chi)=$ \{modular forms (not necessarily analytic) of weight $m$ for level $N$ and associated character $\chi\}$ we have:

$$
\left(\left.G^{*}\right|_{m-(k-2), \chi} \gamma\right)(z)=G^{*}(z)+r_{f}(\gamma)(z) G(z)
$$


or, in a more compact notation,

$$
\left.G^{*}\right|_{m-(k-2), \chi}(\gamma-1)=r_{f}(\gamma) \cdot G \quad \text { for all } \gamma \in \Gamma_{0}(N), z \in \mathfrak{H} .
$$

As usual the 'slash' operator $\left.\right|_{n, \chi}$ is defined by the formula

$$
\left.\left.Q\right|_{n, \chi}\left(\begin{array}{cc}
* & * \\
c & d
\end{array}\right)\right)(z):=\bar{\chi}\left(\left(\begin{array}{cc}
* & * \\
c & d
\end{array}\right)\right) Q(\gamma z)(c z+d)^{-n} \operatorname{det}\left(\left(\begin{array}{cc}
* & * \\
c & d
\end{array}\right)\right)^{n / 2}
$$

and when $\chi$ is trivial we write $\left.\right|_{n}$. Each of these functions can be expressed essentially as a sum of Poincaré series shifted by a modular symbol (cf. [G1]).

In this work, we introduce Hecke operators on series formed with modular symbols which are natural with respect to the structure of the spaces these functions comprise and we study their effect on several associated objects.

In section 2 we view series formed with modular symbols as a kind of generalized modular integral and we apply Knopp's Hecke operators for modular integrals, denoted by $T_{p}$ with $(p, N)=1$, to them. For holomorphic series formed with modular symbols $G^{*}$ that vanish at the cusps, we describe the effect of the operators $T_{p}$ on a certain family of polynomials and demonstrate a relation with values of $L$-functions of $G^{*}$. This is analogous to the relation of the period polynomial to values of $L$-functions of the usual cusp forms.

In sections 3 and 4 the action of the Hecke operators on non-holomorphic Eisenstein series formed with modular symbols, i.e.

$$
E^{*}(z, s ; f)=E^{*}(z, s):=\sum_{\tau \in \Gamma_{\infty} \backslash \Gamma} r_{f}(\tau)(z) \operatorname{Im}(\tau z)^{s},
$$

is examined in detail. A description of the basic properties of these functions (for $f \in S_{2}(M)$ and holomorphic), including their meromorphic continuation, functional equation, poles and residues, was initiated in [G1], and completed by the second author in [O'S1] using a generalization of Selberg's method for the analytic continuation of Eisenstein series. These results have been used by Goldfeld to prove some first theorems on the distribution of modular symbols (cf. [G2]). In this case, the effect of the Hecke operators $U_{q}$ with $q \mid N$, (in the notation of Atkin and Lehner $[\mathrm{AL}])$ on $E^{*}$ yields an interesting relation involving Rankin-Selberg $L$-functions when we consider the action on the residues of the poles of $E^{*}$. As an application of this an orthonormal basis for the spaces of oldforms and newforms coming from a single Maass form on the full modular group is constructed.

In Section 5, some alternative Hecke operators are given which are compatible with different definitions of the space in which our functions $G^{*}$ can be thought of as belonging. Although these operators do not behave as naturally with respect to the analogue of the period polynomial as the first one discussed here, they are, in some cases, better suited to carrying over to $G^{*}$ the eigenproperties of the cusp form to which the modular symbol is associated. Thus, depending on the applications, either type of Hecke operator may be used.

Finally, we should remark that our work can also be understood in terms of a more general project attempting a study of objects associated to modular forms, such as $L$ functions, periods etc., using the action of the relevant group by $\left.\right|_{k}$ for varying index $k$ 
and, consequently, the corresponding cohomology. This viewpoint has already been applied by the first author in the investigation of the derivatives of $L$-functions of (usual) cusp forms (cf. [D1], [D2]) and earlier, by other authors, in the study of modular integrals, rational period functions etc. (cf. [K], [A], [CZ], [G3] etc.)

The authors would like to thank D. Goldfeld, S. Wolpert and D. Zagier for their helpful suggestions and advice in the writing of this paper. This work was completed while the first author was a guest at Max-Planck-Institut für Mathematik in Bonn in the framework of the program Training and Mobility of Researchers.

\section{Hecke operators and $L$-functions.}

We recall that a rational period function of weight $k>0$ for $S L_{2}(\mathbb{Z})$ is a rational function $q$ such that:

$$
q+\left.q\right|_{k} T=q+\left.q\right|_{k} U+\left.q\right|_{k} U^{2}=0 \quad \text { where } \quad T=\left(\begin{array}{cc}
0 & -1 \\
1 & 0
\end{array}\right), U=\left(\begin{array}{cc}
1 & -1 \\
1 & 0
\end{array}\right) .
$$

Also, a modular integral of weight $k$ is a meromorphic function $F$ on the upper-half plane $\mathfrak{H}$ of polynomial growth at the cusps such that: $\left.F\right|_{k} S=F$ and $\left.F\right|_{k} T=F+q$ for $q$ a rational function and $S:=\left(\begin{array}{ll}1 & 1 \\ 0 & 1\end{array}\right)$. It is easy to see that $q$ will then be a period function.

Because of (1), in the special case that $N=1$ the functions defined in the Introduction can be considered as modular integrals associated to period functions which are no longer rational but rather smooth with polynomial growth at the cusps. Motivated by this observation we study first the effect Knopp's Hecke operators for modular integrals (see $[\mathrm{K}]$ ) have on our functions.

With the notation used in the Introduction, we set $n:=m-(k-2)$ and consider the space $\mathfrak{N}$ of smooth functions on $\mathfrak{H}$ with polynomial growth at the cusps. We let $\Gamma_{0}(N)$ act on $\mathfrak{N}$ from the right by $\left.\right|_{n}$ and we denote by $d$ the coboundary operator from the 0-cochains to the 1-cochains (with respect to the 'bar'-resolution). Knopp's Hecke operators on modular integrals $T_{p}$ for $(p, N)=1$, in the formulation of [CZ], is given by the element of $\mathbb{Z}\left[M_{p}\right]$ (where $\left.M_{l}:=\left\{M \in M_{2}(\mathbb{Z}) ; \operatorname{det}(M)=l\right\}\right)$ :

$$
T_{p}:=\sum_{M \in \Delta_{p}} M \quad \text { where } \Delta_{p}:=\left\{\left(\begin{array}{ll}
a & b \\
0 & d
\end{array}\right): a d=p, a>0,0 \leq b<d\right\} .
$$

Thus we set:

$$
T_{p} G^{*}:=G^{*}{ }_{n} T_{p}
$$

Since $d T_{p}=T_{p} d$, where the $T_{p}$ on the right denotes the Hecke operator on 1-cocycles (cf. [S], Ch. 8), we have

$$
\left.\left(T_{p} G^{*}\right)\right|_{n}(\gamma-1)=T_{p}\left(r_{f} \cdot G\right)(\gamma) \text { for all } \gamma \in \Gamma_{0}(N)
$$

We return to the case $N=1$. Since $r_{f}(S) \cdot G=0$, the fact that $r_{f}(\gamma) \cdot G \in \mathfrak{N}$ satisfies a 1-cocycle condition is equivalent to $r_{f}:=r_{f}(T) \cdot G \in \mathfrak{N}$ being annihilated by $T+1$ and $U^{2}+U+1$. Using Knopp's Hecke operators as described in [CZ], we can give an explicit formula for the period function associated to $T_{p} G^{*}$ in terms of $r_{f}$. 
Proposition $1[\mathrm{CZ}]$. Let $T_{p}$ and $M_{l}$ be as above and let $J$ denote the right ideal $(1+T) \mathbb{Z}\left[M_{1}\right]+\left(1+U+U^{2}\right) \mathbb{Z}\left[M_{1}\right]$ of $\mathbb{Z}\left[M_{1}\right]$. Then, there are $X_{p}, Y_{p} \in \mathbb{Z}\left[M_{p}\right]$ such that (i) $X_{p} J \subset J \mathbb{Z}\left[M_{p}\right]$ and $(i i) T_{p}(T-1)=(T-1) X_{p}+(S-1) Y_{p}$. Moreover an example of such an $X_{p}$ is given, namely $X_{p}:=\sum_{M \in \mathfrak{M}} M$ where $\mathfrak{M}=\left\{\left(\begin{array}{ll}a & b \\ c & d\end{array}\right) \in M_{p}\right.$ such that $a>|c|, d>|b|, b c \leq 0, c=0 \Longrightarrow-d / 2<b \leq d / 2$, and $b=0 \Longrightarrow-a / 2<c \leq$ $a / 2\}$.

In a manner completely analogous to the application of that theorem discussed in [CZ], we have:

$\left.\left(T_{p} G^{*}\right)\right|_{n}(T-1)=\left.\left(G^{*}{ }_{n} T_{p}\right)\right|_{n}(T-1)=\left.\left.G^{*}\right|_{n}(T-1)\right|_{n} X_{p}+\left.\left.G^{*}\right|_{n}(S-1)\right|_{n} Y_{p}=\left.r_{f}\right|_{n} X_{p}$ (since, as it is easy to see, $\left.G^{*}\right|_{n} S=G^{*}$ ) and because of part (i) of the theorem, $\left.r_{f}\right|_{n} X_{p}$ is a period function too.

We study next how these Hecke operators interact with the analogue of period polynomials for those of our functions which are holomorphic and vanish at the cusps (see [G1]). To do that we quickly recall the definition of the $L$-function associated to series formed with modular symbols $G^{*}$ (see [G1]).

Let $G^{*}$ have a Fourier expansion (at $i \infty$ ) of the form $G^{*}(z)=\sum_{n=1}^{\infty} a(n) e(n z)$. In [G1] it is proven that

$$
L_{G^{*}}(s):=\sum_{n=0}^{\infty} \frac{a(n)}{n^{s}}
$$

converges absolutely for $s$ in some right half-plane and that it can be expressed in terms of the inverse Mellin transform:

$$
\int_{0}^{\infty} G^{*}(i y) y^{s-1} d y=\Gamma(s)(2 \pi)^{-s} L_{G^{*}}(s) .
$$

The proof is identical with that of the case of cusp forms because the key property used is the exponential decay at the cusps which holds also for $G^{*}$. This equation enables us to extend $L_{G^{*}}$ to an entire function. More generally, the $L$-function of $G^{*}$ twisted by an exponential character can be expressed in terms of integrals of the form $\int_{x}^{\infty} G^{*}(i y)(y-x)^{s-1} d y$ with $x \in \mathbb{Q}$.

It is then natural to define the map sending an element $\gamma$ of $\Gamma_{0}(N)$ to the polynomial:

$$
\sigma(\gamma):=\int_{i \infty}^{\gamma^{-1} i \infty} G^{*}(z)(z-X)^{n-2} d z
$$

where $n=m-(k-2) \geq 2$. When $N=1$, the coefficients of $\sigma(T)$ are explicit linear combinations of values of $L_{G^{*}}(s)$ inside the critical strip (as is the case for the period polynomial as well).

Proposition 2. Let $G^{*}$ be a holomorphic function on $\mathfrak{H}$ which vanishes at the cusps and satisfies equation (1) for some $f \in S_{k}(1)$ and $G \in S_{m}(1)$. Then,

$$
\sigma(T)=\sum_{j=0}^{n-2}\left(\begin{array}{c}
n-2 \\
j
\end{array}\right)(-1)^{j-1} j !\left(\frac{i}{2 \pi}\right)^{j+1} L_{G^{*}}(j+1) X^{n-2-j} .
$$




\section{Proof.}

By the definition (and binomial expansion):

$$
\sigma(T)=\sum_{j=0}^{n-2}\left(\begin{array}{c}
n-2 \\
j
\end{array}\right)(-1)^{j}\left(\int_{i \infty}^{0} G^{*}(z) z^{j} d z\right) X^{n-2-j}
$$

The result is obtained by this formula and the expression of $L_{G}^{*}$ in terms of the inverse Mellin transform for $s=j+1$.

More generally, the coefficients of $\sigma(g)$, for any $g \in \Gamma_{0}(N)$ are linear combinations of values of the twisted (by an exponential) $L$-function. The map satisfies a 1-cocycle condition with respect to the usual action $\left.\right|_{2-n}$ of $\Gamma_{0}(N)$ on the polynomials of degree $\leq n-2$ "modulo linear combinations of products of periods of $f$ and $G$ ". We recall that the period of a weight $k$ cusp form $g$ for $\Gamma_{0}(N)$ is an integral $\int_{0}^{i \infty} g(z) z^{j} d z$ for $j \in\{0, \ldots, k-2\}$.

Specifically,

Proposition 3. For all $\gamma_{1}, \gamma_{2} \in \Gamma_{0}(N)$ we have:

$$
\sigma\left(\gamma_{2} \gamma_{1}\right)=\left.\sigma\left(\gamma_{2}\right)\right|_{2-n} \gamma_{1}+\sigma\left(\gamma_{1}\right)+\left.\left(\int_{i \infty}^{\gamma_{2}^{-1} i \infty} r_{f}\left(\gamma_{1}^{-1}\right)(z) G(z)(z-X)^{n-2} d z\right)\right|_{2-n} \gamma_{1}
$$

\section{Proof.}

By the equality $\left(z-\gamma_{1} X\right) j\left(\gamma_{1}, X\right)=\left(\gamma_{1}^{-1} z-X\right) j\left(\gamma_{1}^{-1}, z\right)$ and equality (1), we obtain:

$$
\begin{aligned}
\left.\sigma\left(\gamma_{2}\right)\right|_{2-n} \gamma_{1} & =\int_{i \infty}^{\gamma_{2}^{-1} i \infty} G^{*}(z)\left(z-\gamma_{1} X\right)^{n-2} j\left(\gamma_{1}, X\right)^{n-2} d z \\
& =\int_{i \infty}^{\gamma_{2}^{-1} i \infty} G^{*}\left(\gamma_{1}^{-1} z\right)\left(\gamma_{1}^{-1} z-X\right)^{n-2} d\left(\gamma_{1}^{-1} z\right) \\
& -\int_{i \infty}^{\gamma_{2}^{-1} i \infty} r_{f}\left(\gamma_{1}^{-1}\right)(z) G(z)\left(\gamma_{1}^{-1} z-X\right)^{n-2} j\left(\gamma_{1}^{-1}, z\right)^{n-2} d z
\end{aligned}
$$

By a change of variables, we observe that the first integral equals $\sigma\left(\gamma_{2} \gamma_{1}\right)-\sigma\left(\gamma_{1}\right)$ and this completes the proof.

This formula implies that $\sigma$ is determined by its values at the generators of $\Gamma_{0}(N)$ and by the (usual) periods of $G$ and $f$. In particular, for $N=1$, it is determined ("modulo products of usual periods") by its value at $T$, ( $\operatorname{since} \sigma\left(\left(\begin{array}{ll}1 & 1 \\ 0 & 1\end{array}\right)\right)=0$ ), so this value can be considered as the analogue of the period polynomial.

In order to characterize the effect of the Hecke operator of $G^{*}$ on the associated map $\sigma$, we need a easy generalization of Proposition 1 for $\Gamma_{0}(N)(N \geq 1)$. 
Lemma 4. Let $\left\{\gamma_{1}, \ldots, \gamma_{r}\right\}$ be a set of generators of $\Gamma_{0}(N)$. For every $\gamma \in \Gamma_{0}(N)$ there are $X_{1}, \ldots, X_{r} \in \mathbb{Z}\left[M_{p}\right]$ such that

$$
T_{p}(\gamma-1)=\left(\gamma_{1}-1\right) X_{1}+\cdots+\left(\gamma_{r}-1\right) X_{r}
$$

\section{Proof.}

For each $M \in \Delta_{p}$, there is a $\gamma_{M} \in \Gamma_{0}(N)$ and a (unique) $\hat{M} \in \Delta_{p}$ such that $M \gamma \hat{M}^{-1}=\gamma_{M}$ (cf. [S], Prop. 3.36). Therefore, $T_{p}(\gamma-1)=\sum_{M}\left(\gamma_{M} \hat{M}-M\right)=$ $\sum_{M}\left(\gamma_{M}-1\right) \hat{M}$. The lemma follows by this equality and the observation that, for all $\gamma \in \Gamma_{0}(N), \gamma-1 \in\left(\gamma_{1}-1\right) \mathbb{Z}\left[M_{1}\right]+\cdots+\left(\gamma_{r}-1\right) \mathbb{Z}\left[M_{1}\right]$. (If $\gamma$ satisfies this property, then for $i=1, \ldots, r, \gamma_{i} \gamma-1=\left(\gamma_{i}-1\right) \gamma+(\gamma-1), \gamma_{i}^{-1} \gamma-1=\left(\gamma_{i}-1\right)\left(-\gamma_{i}^{-1} \gamma\right)+(\gamma-1)$ and the fact follows by induction.)

Theorem 5. With the above notation, let $G^{*}$ be a holomorphic function on $\mathfrak{H}$ which vanishes at the cusps and satisfies equation (1) for some $f \in S_{k}(M)$ and $G \in S_{m}(N)$. Then, for all $\gamma \in \Gamma_{0}(N)$ and $p$ such that $(p, N)=1$ we have,

$$
\sigma_{T_{p} G^{*}}(\gamma)=\left.\sum_{i} \sigma_{G^{*}}\left(\gamma_{i}\right)\right|_{2-n} X_{i}+P
$$

where $n:=m-(k-2)$ and $P$ is a polynomial of degree $\leq n-2$ whose coefficients are rational combinations of products of periods of $G$ and $f$.

\section{Proof.}

We set $s_{G^{*}}(\tau)=\int_{\tau}^{i \infty} G^{*}(z)(z-\tau)^{n-2} d z$. Then, we have

$$
\left(\left.s_{G^{*}}\right|_{2-n}(\gamma-1)\right)(\tau)=\int_{\gamma \tau}^{\gamma\left(\gamma^{-1} i \infty\right)} G^{*}(z)(z-\gamma \tau)^{n-2} j(\gamma, \tau)^{n-2} d z-s_{G^{*}}(\tau),
$$

and this, by a change of variables in the first integral, equals

$$
\begin{aligned}
\int_{\tau}^{\gamma^{-1}(i \infty)}\left(\left.G^{*}\right|_{n} \gamma\right)(z) & (z-\tau)^{n-2} d z-\int_{\tau}^{i \infty} G^{*}(z)(z-\tau)^{n-2} d z \\
& =\sigma_{G^{*}}(\gamma)+\int_{\tau}^{\gamma^{-1}(i \infty)} r_{f}(\gamma)(z) G(z)(z-\tau)^{n-2} d z
\end{aligned}
$$

because $(\gamma z-\gamma \tau) j(\gamma, z) j(\gamma, \tau)=z-\tau$. Since $T_{p}\left(r_{f} \cdot G\right)(\gamma)$ is the period function corresponding to $T_{p} G^{*}$, this also implies:

$$
\left(\left.s_{T_{p} G^{*}}\right|_{2-n}(\gamma-1)\right)(\tau)=\sigma_{T_{p} G^{*}}(\gamma)+\int_{\tau}^{\gamma^{-1}(i \infty)} T_{p}\left(r_{f} \cdot G\right)(\gamma)(z)(z-\tau)^{n-2} d z .
$$

Moreover, by Lemma 4, we have that

$$
\begin{aligned}
T_{p}\left(r_{f} G\right)(\gamma)=\left.\left(T_{p} G^{*}\right)\right|_{n}(\gamma-1) & =\left.\left.G^{*}\right|_{n} T_{p}\right|_{n}(\gamma-1) \\
& =\left.\sum_{i} G^{*}\right|_{n}\left(\left(\gamma_{i}-1\right) \cdot X_{i}\right)=\left.\sum_{i}\left(r_{f}\left(\gamma_{i}\right) \cdot G\right)\right|_{n} X_{i}
\end{aligned}
$$


Hence equality (2) can be written in the form:

$$
\left(\left.s_{T_{p} G^{*}}\right|_{2-n}(\gamma-1)\right)(\tau)=\sigma_{T_{p} G^{*}}(\gamma)+\sum_{i} \int_{\tau}^{\gamma^{-1}(i \infty)}\left(\left.\left(r_{f}\left(\gamma_{i}\right) \cdot G\right)\right|_{n} X_{i}\right)(z)(z-\tau)^{n-2} d z
$$

By definition,

$$
s_{T_{p} G^{*}}(\tau)=p^{n / 2} \sum_{i=0}^{p} \int_{\tau}^{i \infty} G^{*}\left(\alpha_{i} z\right) j\left(\alpha_{i}, z\right)^{-n}(z-\tau)^{n-2} d z .
$$

However, for all $M \in G L_{2}(\mathbb{R})$,

$$
(z-M \tau) j(M, \tau)=\operatorname{det}(M)\left(M^{-1} z-\tau\right) j\left(M, M^{-1} z\right)^{-1} \text { and } d\left(M^{-1} z\right)=\frac{\operatorname{det}\left(M^{-1}\right) d z}{j\left(M^{-1}, z\right)^{2}}
$$

so, by a change of variables in each of the integrals, we eventually see that,

$$
s_{T_{p} G^{*}}(\tau)=p^{n / 2-1-(n-2)} \sum_{i=0}^{p} \int_{\alpha_{i} \tau}^{i \infty} G^{*}(z) j\left(\alpha_{i}, z\right)^{n-2}\left(z-\alpha_{i} \tau\right)^{n-2} d z=\left(\left.s_{G^{*}}\right|_{2-n} T_{p}\right)(\tau) .
$$

Putting all these equalities together, we obtain:

$$
\begin{aligned}
& \sigma_{T_{p} G^{*}}(\gamma)=\left(\left.s_{T_{p} G^{*}}\right|_{2-n}(\gamma-1)\right)(\tau)-\sum_{i} \int_{\tau}^{\gamma^{-1} i \infty}\left(\left.\left(r_{f}\left(\gamma_{i}\right) \cdot G\right)\right|_{n} X_{i}\right)(z)(z-\tau)^{n-2} d z \\
& =\left(\left.s_{G^{*}}\right|_{2-n} T_{p}(\gamma-1)\right)(\tau)-\sum_{i} \int_{\tau}^{\gamma^{-1}(i \infty)}\left(\left.\left(r_{f}\left(\gamma_{i}\right) \cdot G\right)\right|_{n} X_{i}\right)(z)(z-\tau)^{n-2} d z .
\end{aligned}
$$

Using Lemma 4 once more, the last sum becomes:

$$
\begin{gathered}
\sum_{i=1}^{r}\left(\left.\left.s_{G^{*}}\right|_{2-n}\left(\gamma_{i}-1\right)\right|_{2-n} X_{i}\right)(\tau)-\sum_{i} \int_{\tau}^{\gamma^{-1} i \infty}\left(\left.\left(r_{f}\left(\gamma_{i}\right) \cdot G\right)\right|_{n} X_{i}\right)(z)(z-\tau)^{n-2} d z \text { or } \\
\left.\sum_{i=1}^{r} \sigma_{G^{*}}\left(\gamma_{i}\right)\right|_{2-n} X_{i}+\left.\sum_{i}\left(\int_{\tau}^{\gamma_{i}^{-1} i \infty} r_{f}\left(\gamma_{i}\right)(z) G(z)(z-\tau)^{n-2} d z\right)\right|_{2-n} X_{i} \\
-\sum_{i} \int_{\tau}^{\gamma^{-1}(i \infty)}\left(\left.\left(r_{f}\left(\gamma_{i}\right) \cdot G\right)\right|_{n} X_{i}\right)(z)(z-\tau)^{n-2} d z
\end{gathered}
$$

Now we observe that for each $i$, if $X_{i}=\sum M \in \mathbb{Z}\left[M_{p}\right]$, then

$$
\begin{aligned}
\int_{\tau}^{\gamma^{-1}(i \infty)} & \left(\left.\left(r_{f}\left(\gamma_{i}\right) \cdot G\right)\right|_{n} X_{i}\right)(z)(z-\tau)^{n-2} d z= \\
& \sum_{M} \int_{\tau}^{\gamma^{-1} i \infty} r_{f}\left(\gamma_{i}\right)(M z) G(M z)(z-\tau)^{n-2} j(M, z)^{-n} p^{\frac{n}{2}} d z .
\end{aligned}
$$


Because of (3), this equals,

$$
\sum_{M} p^{1-\frac{n}{2}} \int_{\tau}^{\gamma^{-1} i \infty} r_{f}\left(\gamma_{i}\right)(M z) G(M z)(M z-M \tau)^{n-2} j(M, \tau)^{n-2} d(M z)
$$

or, by a change of variables,

$$
\left.\sum_{M}\left(\int_{\tau}^{M \gamma^{-1} i \infty} r_{f}\left(\gamma_{i}\right)(z) G(z)(z-\tau)^{n-2} d z\right)\right|_{2-n} M
$$

Therefore sum (4) equals,

$$
\left.\sum_{i=1}^{r} \sigma_{G^{*}}\left(\gamma_{i}\right)\right|_{2-n} X_{i}+\left.\sum_{i=1}^{r} \sum_{M}\left(\int_{M \gamma^{-1} i \infty}^{\gamma_{i}^{-1} i \infty} r_{f}\left(\gamma_{i}\right)(z) G(z)(z-\tau)^{n-2} d z\right)\right|_{2-n} M .
$$

Since $r_{f}(\gamma)(z) G(z)=\sum_{j=0}^{k-2}\left(\begin{array}{c}k-2 \\ j\end{array}\right)(-1)^{j} z^{j} G(z) \int_{i \infty}^{\gamma^{-1} i \infty} f(w) w^{k-2-j} d w$ the double sum is a polynomial whose coefficients are linear combinations of products of the form: $\left(\int_{\alpha}^{\beta} G(z) z^{i} d z\right)\left(\int_{i \infty}^{\gamma^{-1} i \infty} f(w) z^{j} d z\right)$ with $0 \leq i \leq n-2+k-2=m-2$ and $0 \leq j \leq k-2$ and $\alpha, \beta \in \mathbb{Q}$. Such integrals can be written as rational linear combinations of periods (cf. [M]). From this the desired result follows.

Corollary 6. Let $f \in S_{k}\left(S L_{2}(\mathbb{Z})\right)$ and $G \in S_{m}\left(S L_{2}(\mathbb{Z})\right)$. Then we have,

$$
\sigma_{T_{p} G^{*}}(T)=\left.\sigma_{G^{*}}(T)\right|_{n} X_{p}+P
$$

where $n:=m-(k-2)$ and $P$ is a polynomial of degree $\leq n-2$ whose coefficients are rational combinations of products of periods of $G$ and $f$.

\section{Proof.}

Since $\sigma_{G^{*}}(S)=0$, this follows from the proof of Theorem 5 (for $\gamma=T$ ) where rather than using Lemma 4 we use Proposition 1.

The same results hold for primes $p$ for $p \mid N$ with $T_{p}$ replaced by the operator $U_{p}:=\sum_{i=0}^{p-1}\left(\begin{array}{ll}1 & i \\ 0 & p\end{array}\right)$. The reason for this is that (as in the case of $\left.T_{p}\right)$ for all $\gamma \in \Gamma_{0}(N)$ and $i=0, \ldots, p-1$, there is a unique $j \in\{0, \ldots, p-1\}$, such that $\left(\begin{array}{ll}1 & i \\ 0 & p\end{array}\right) \gamma\left(\begin{array}{ll}1 & j \\ 0 & p\end{array}\right)^{-1} \in$ $\Gamma_{0}(N)$.

\section{Eisenstein series formed with modular symbols and the operators $T_{p}$.}

Let $\Gamma=\Gamma_{0}(N)$ be the Hecke congruence group of level $N$. In [G1], [O'S] the non-holomorphic Eisenstein series

$$
E^{*}(z, s ; f)=E^{*}(z, s):=\sum_{\tau \in \Gamma_{\infty} \backslash \Gamma} r_{f}(\tau)(z) \operatorname{Im}(\tau z)^{s}
$$


is studied in the special case that $f$ is a weight 2 holomorphic cusp form. In that case $r_{f}(\tau)(z)=r_{f}(\tau)=\langle\tau, f\rangle /(2 \pi i)$ is a modular symbol. As can be readily verified, for a fixed $s, E^{*}(\cdot, s)$ satisfies (1) with $\chi=1, m=k-2=0$ and $G(z)=E(z, s)=$ $\sum_{\tau \in \Gamma_{\infty} \backslash \Gamma} \operatorname{Im}(\tau z)^{s}$, the usual automorphic non-holomorphic Eisenstein series. The series (5) converges to a holomorphic function of $s$ for $\operatorname{Re}(s)>2$. As a function of $z$ it is an eigenfunction of the hyperbolic Laplacian $\Delta=-y^{2}\left(\frac{\partial^{2}}{\partial x^{2}}+\frac{\partial^{2}}{\partial y^{2}}\right)$ and is real-analytic. In [O'S2] it is shown that $E^{*}$ has a meromorphic continuation to the entire $s$-plane and a functional equation relating values at $s$ to those at $1-s$. In [O'S1] and work to appear it is also shown that $E^{*}$ has simple poles on the critical line $\operatorname{Re}(s)=1 / 2$.

In this section we examine the effect the Hecke operators have on such series and derive a relation for the values of certain convolution $L$-functions at special points. We fix, once and for all, a weight 2 holomorphic newform $f(z)$ for $\Gamma$. This implies that $f$ is an eigenfunction of the Hecke operators $T_{p}$ for primes $p \nmid N$ and $U_{q}$ for primes $q \mid N$. If $f$ has Fourier expansion

$$
f(z)=\sum_{n=1}^{\infty} a(n) e(n z)
$$

then we may normalize $f$ to have $a(1)=1$. In that case $T_{p} f=a(p) f$ and $U_{q} f=a(q) f$. As is well known, the coefficients $a(n)$ have the following multiplicative properties:

$$
\begin{aligned}
a\left(p^{r}\right) a(p) & =a\left(p^{r+1}\right)+a\left(p^{r-1}\right) p \text { if } p \nmid N, \\
a\left(p^{r}\right) & =a(p)^{r} \text { if } p \mid N, \\
\text { and } a(m) a(n) & =a(m n) \text { if }(m, n)=1 .
\end{aligned}
$$

Thus in particular we have the formula

$$
a(p n)=a(p) a(n)-\left\{\begin{array}{rl}
a(n / p) p & \text { if } p \mid n \text { and } p \nmid N \\
0 & \text { if } p \nmid n \text { or } p \mid N
\end{array} .\right.
$$

Also it is known that $a(p)=0$ if $p^{2} \mid N$ and $a(p)= \pm 1$ if $p \mid N$ and $p^{2} \nmid N$. We next set

$$
F_{f}(z)=F(z)=\int_{i \infty}^{z} f(w) d w,
$$

the antiderivative of $f$. The Hecke operators act naturally on the 'automorphic part' of our function

$$
Q^{*}(z, s ; f)=\sum_{\tau \in \Gamma_{\infty} \backslash \Gamma} F(\tau z) \operatorname{Im}(\tau z)^{s}=E^{*}(z, s)+F(z) E(z, s),
$$

where it is clear that $Q^{*}$ is automorphic with weight zero. To prove the proposition describing the effect of $T_{p}$ on $Q^{*}$, we need the following two lemmas. 
Lemma 7. For each prime $p$ such that $p \nmid N$ we have $T_{p} F:=\left.F\right|_{0} T_{p}=a(p) F$.

\section{Proof.}

$$
\begin{aligned}
T_{p} F(z) & =\sum_{\rho \in \Delta_{p}} \int_{i \infty}^{\rho z} f(w) d w=\sum_{\rho} \int_{\rho i \infty}^{\rho z} f(w) d w \\
& =\sum_{\rho} \int_{i \infty}^{z} f(\rho w) d \rho w=\int_{i \infty}^{z}\left[T_{p} f(w)\right] d w=a(p) F(z) .
\end{aligned}
$$

Note that if $q \mid N$ then $U_{q} F:=\left.F\right|_{0} U_{q}=a(q) F$ by the same proof.

Lemma 8. Let $H_{N}$ be a set of representatives of $\Gamma_{\infty} \backslash \Gamma_{0}(N)$. Then, for each prime $p$ with $(N, p)=1$ we have the equality

$$
\bigcup_{\substack{\rho \in \Delta_{p} \\ \tau \in H_{N}}} \Gamma_{\infty} \tau \rho=\bigcup_{\substack{\rho \in \Delta_{p} \\ \tau \in H_{N}}} \Gamma_{\infty} \rho \tau
$$

where the cosets on the left and right are disjoint.

\section{Proof.}

We first prove that $\bigcup_{\substack{\rho \in \Delta_{p} \\ \tau \in H_{N}}} \Gamma_{\infty} \rho \tau \subset \bigcup_{\substack{\rho \in \Delta_{p} \\ \tau \in H_{N}}} \Gamma_{\infty} \tau \rho$. For each $\tau \in \Gamma_{0}(N)$ there is a unique $\tilde{\rho} \in \Delta_{p}$ such that $\rho \tau \tilde{\rho}^{-1} \in \Gamma_{0}(N)$ (see [S], Prop. 3.36). Then, $\rho \tau \tilde{\rho}^{-1}$ will equal $S^{n} \tau^{\prime}$ for some $n \in \mathbb{Z}, \tau^{\prime} \in H_{N}$, where $S$ is the generator of $\Gamma_{\infty}$, i.e.

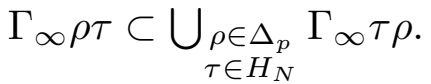

Conversely, let $\tau \in H_{N}$ and $\rho \in \Delta_{p}$. Then there is an $n \in \mathbb{Z}$ and $\tilde{\rho} \in \Delta_{p}$ such that $\tilde{\rho}^{-1} S^{n} \tau \rho \in \Gamma_{0}(N)$. This $n$ can be found as follows. If $p \mid c$, then

$$
\begin{aligned}
\left(\begin{array}{ll}
1 & 0 \\
0 & p
\end{array}\right)^{-1}\left(\begin{array}{ll}
a & b \\
c & d
\end{array}\right)\left(\begin{array}{ll}
1 & i \\
0 & p
\end{array}\right) & =\left(\begin{array}{cc}
a & a i+b p \\
\frac{c}{p} & \frac{c}{p} i+d
\end{array}\right) \in \Gamma_{0}(N) \text { and } \\
\left(\begin{array}{ll}
p & 0 \\
0 & 1
\end{array}\right)^{-1}\left(\begin{array}{ll}
1 & n \\
0 & 1
\end{array}\right)\left(\begin{array}{ll}
a & b \\
c & d
\end{array}\right)\left(\begin{array}{ll}
p & 0 \\
0 & 1
\end{array}\right) & =\left(\begin{array}{cc}
a+n c & \frac{b+n d}{p} \\
p c & d
\end{array}\right) \in \Gamma_{0}(N)
\end{aligned}
$$

for any $n \in \mathbb{Z}$ such that $p \mid(b+n d)$. If $p \nmid c$, then

$$
\left(\begin{array}{ll}
p & 0 \\
0 & 1
\end{array}\right)^{-1}\left(\begin{array}{ll}
1 & n \\
0 & 1
\end{array}\right)\left(\begin{array}{ll}
a & b \\
c & d
\end{array}\right)\left(\begin{array}{ll}
1 & i \\
0 & p
\end{array}\right)=\left(\begin{array}{cc}
\frac{a+n c}{p} & \frac{a+n c}{p} i+b+n d \\
c & c i+d p
\end{array}\right) \in \Gamma_{0}(N)
$$

for $n$ with $p \mid a+n c$ and

$$
\begin{aligned}
\left(\begin{array}{ll}
1 & 0 \\
0 & p
\end{array}\right)^{-1}\left(\begin{array}{ll}
a & b \\
c & d
\end{array}\right)\left(\begin{array}{ll}
p & 0 \\
0 & 1
\end{array}\right) & =\left(\begin{array}{cc}
p a & b \\
p & \frac{d}{p}
\end{array}\right) \in \Gamma_{0}(N), \text { if } p \mid d, \text { or } \\
\left(\begin{array}{ll}
p & 0 \\
0 & 1
\end{array}\right)^{-1}\left(\begin{array}{ll}
1 & n \\
0 & 1
\end{array}\right)\left(\begin{array}{ll}
a & b \\
c & d
\end{array}\right)\left(\begin{array}{ll}
p & 0 \\
0 & 1
\end{array}\right) & =\left(\begin{array}{cc}
a+n c & \frac{b+n d}{p} i+b+n d \\
p c & d
\end{array}\right) \in \Gamma_{0}(N)
\end{aligned}
$$

for any $n$ with $p \mid b+n d$ if $p \nmid d$. Hence, $\Gamma_{\infty} \tau \rho \subset \bigcup_{\substack{\rho \in \Delta_{p} \\ \tau \in H_{N}}} \Gamma_{\infty} \rho \tau$.

We are now ready to prove 
Proposition 9. Set $f_{p}(z):=p f(p z)$ then for each prime $p$ not dividing $N$

$$
T_{p} Q^{*}(z, s)=a(p) p^{-s} Q^{*}(z, s)+\left(p^{s}-p^{-s}\right) Q^{*}\left(z, s ; f_{p}\right) .
$$

\section{Proof.}

$$
T_{p} Q^{*}(z, s)=\sum_{\rho \in \Delta_{p}} \sum_{\tau \in \Gamma_{\infty} \backslash \Gamma} F(\tau \rho z) \operatorname{Im}(\tau \rho z)^{s}=\sum_{\rho} \sum_{\tau} F(\rho \tau z) \operatorname{Im}(\rho \tau z)^{s}
$$

Note that $\operatorname{Im}(\rho z)=\frac{1}{p} \operatorname{Im}(z)$ for each $\rho=\left(\begin{array}{cc}1 & i \\ 0 & p\end{array}\right)$ and $\operatorname{Im}\left(\left(\begin{array}{ll}p & 0 \\ 0 & 1\end{array}\right) z\right)=p \operatorname{Im}(z)$. So we obtain

$$
\begin{aligned}
T_{p} Q^{*}(z, s) & =p^{s} \sum_{\tau}\left(F(p \tau z)+p^{-2 s}\left(\sum_{\rho} F(\rho \tau z)-F(p \tau z)\right)\right) \operatorname{Im}(\tau z)^{s} \\
& =p^{s} \sum_{\tau}\left(F(p \tau z)\left(1-p^{-2 s}\right)+p^{-2 s} \sum_{\rho} F(\rho \tau z)\right) \operatorname{Im}(\tau z)^{s} \\
& =p^{s} \sum_{\tau}\left(F(p \tau z)\left(1-p^{-2 s}\right)+p^{-2 s} a(p) F(\tau z)\right) \operatorname{Im}(\tau z)^{s} \\
& =\left(p^{s}-p^{-s}\right) \sum_{\tau} F(p \tau z) \operatorname{Im}(\tau z)^{s}+a(p) p^{-s} Q^{*}(z, s) .
\end{aligned}
$$

Noting that

$$
\int_{i \infty}^{z} p f(p w) d w=\int_{i \infty}^{p z} f(w) d w=F(p z)
$$

completes the proof.

As we remarked earlier $E^{*}$ has simple poles at certain points $s^{\prime}$ on the critical line $\operatorname{Re}(s)=1 / 2$. We wish to examine the effect of the Hecke operators on the residues of these poles.

Let $L^{2}(\Gamma \backslash \mathfrak{H})$ denote the space of automorphic (weight zero) functions $\phi$ on $\Gamma \backslash \mathfrak{H}$ with finite norm $\langle\phi, \phi\rangle_{N}$ where the Petersson inner product is given by

$$
\langle\phi, \psi\rangle_{N}=\int_{\Gamma_{0}(N) \backslash \mathfrak{H}} \phi(z) \overline{\psi(z)} d \mu z,
$$

for $z=x+i y$ and $d \mu z=d x d y / y^{2}$. The Laplacian induces the spectral decomposition

$$
L^{2}(\Gamma \backslash \mathfrak{H})=\mathbb{C} \oplus \mathcal{C}(\Gamma \backslash \mathfrak{H}) \oplus \mathcal{E}(\Gamma \backslash \mathfrak{H})
$$

where $\mathbb{C}$ is the space of constant functions, $\mathcal{C}$ the space of cusp forms and $\mathcal{E}$ the space of Eisenstein series. Let $\eta_{j}$, for $j=0,1,2 \ldots$, be an orthonormal basis for $\mathbb{C} \oplus \mathcal{C}(\Gamma \backslash \mathfrak{H})$ 
with $\Delta \eta_{j}=\lambda_{j} \eta_{j}$ and $0=\lambda_{0}<\lambda_{1} \leq \lambda_{2} \leq \ldots$. We may assume that the Maass cusp forms $\eta_{j}$ with $j \geq 1$ satisfy $T_{n} \eta_{j}(z)=\lambda_{j}(n) \eta_{j}(z)$ for all $n$ with $(n, N)=1$. These Maass forms have Fourier expansion

$$
\eta_{j}(z)=\sum_{n \neq 0} b_{j}(n) W_{s}(n z)
$$

where $W$ is the Whittaker function, see $[\mathrm{Iw}]$. Similarly to (7) we have, for $p$ prime not dividing $N$,

$$
b_{j}(p n) p=\lambda_{j}(p) b_{j}(n)-\left\{\begin{array}{rl}
b_{j}(n / p) & \text { if } p \mid n \\
0 & \text { if } p \nmid n
\end{array} .\right.
$$

If, for example, $\eta_{j}$ is a newform with $b_{j}(1) \neq 0$ then $\lambda_{j}(p) / p=b_{j}(p) / b_{j}(1)$.

In [O'S] it is shown that $E^{*}(z, s)$ has simple poles at points $s_{j}$ satisfying $s_{j}\left(1-s_{j}\right)=$ $\lambda_{j}$. The residue at such a point $s^{\prime}$ is given by

$$
\frac{\pi^{\frac{1}{2}-s^{\prime}}}{4 \pi i} \Gamma\left(s^{\prime}-1 / 2\right) \sum_{j: s_{j}=s^{\prime}} L_{f \otimes \eta_{j}}\left(s^{\prime}\right) \eta_{j}(z),
$$

provided this is non-zero. For the remainder we may ignore the non-zero factor in front of the sum. The convolution $L$-function with $f$ and $\eta_{j}$ as in (6) and (8) has definition

$$
L_{f \otimes \eta_{j}}(s)=\sum_{n=1}^{\infty} \frac{a(n) \overline{b_{j}(n)}}{n^{s}} .
$$

Also known as Rankin-Selberg zeta functions they have been studied in connection with the disappearance of Maass cusp forms $\eta_{j}(z)$ for $\Gamma_{0}(N)$ when the group is subject to a quasi-conformal deformation determined by a holomorphic cusp form $f(z)$. In [PS] it is shown that $\eta_{j}$ is annihilated if and only if $L_{f \otimes \eta_{j}}\left(s_{j}\right) \neq 0$. In further work $[\mathrm{DI}]$ and $[\mathrm{L}]$ prove that a high proportion of these zeta functions are non-zero at this special point. If $s_{j}=1 / 2+i t_{j}$, it is known that $\#\left\{j:\left|t_{j}\right| \leq T\right\} \sim C T^{2}$ for a constant $C$ and in $[\mathrm{L}]$ the estimate

$$
\#\left\{j:\left|t_{j}\right| \leq T, L_{f \otimes \eta_{j}}\left(s_{j}\right) \neq 0\right\} \gg T^{2-\epsilon}
$$

is proven.

These results indicate that Maass cusp forms are rarer than previously thought. It also shows that $E^{*}$ has infinitely many poles on the critical line. Thus the value of $L_{f \otimes \eta_{j}}\left(s_{j}\right)$ appearing naturally in (10) is of great significance.

Continuing our analysis, since the difference $E^{*}-Q^{*}=F E$ is holomorphic for $\operatorname{Re}(s) \geq 1 / 2$ except at $s=1$ we can work instead with $Q^{*}$. We see that the operators $T_{p}$ act on $\operatorname{Res}_{s=s_{j}} Q^{*}(z, s)$ in two ways, directly on the Maass forms and also on $Q^{*}$ :

$$
T_{p} \operatorname{Res}_{s=s^{\prime}} Q^{*}(z, s)=\operatorname{Res}_{s=s^{\prime}} T_{p} Q^{*}(z, s) .
$$


Thus, for $(p, N)=1$,

$$
\begin{aligned}
\sum_{j: s_{j}=s^{\prime}} L_{f \otimes \eta_{j}}\left(s^{\prime}\right) \lambda_{j}(p) \eta_{j}(z)=a(p) p^{-s^{\prime}} \sum_{j: s_{j}=s^{\prime}} L_{f \otimes \eta_{j}}\left(s^{\prime}\right) \eta_{j}(z) & \\
& +\left(p^{s^{\prime}}-p^{-s^{\prime}}\right) \sum_{j: s_{j}=s^{\prime}} L_{f_{p} \otimes \eta_{j}}\left(s^{\prime}\right) \eta_{j}(z),
\end{aligned}
$$

implying that

$$
\left(\lambda_{j}(p)-a(p) p^{-s_{j}}\right) L_{f \otimes \eta_{j}}\left(s_{j}\right)=\left(p^{s_{j}}-p^{-s_{j}}\right) L_{f_{p} \otimes \eta_{j}}\left(s_{j}\right)
$$

This formula is actually true for all $s$ not just $s=s_{j}$ as the next proposition shows.

Proposition 10. For any Maass form $\eta_{j}$ as above and any $s \in \mathbb{C}$ we have

$$
\left(\lambda_{j}(p)-a(p) p^{-s}\right) L_{f \otimes \eta_{j}}(s)=\left(p^{s}-p^{-s}\right) L_{f_{p} \otimes \eta_{j}}(s)
$$

for $f_{p}(z)=p f(p z)$ and $(p, N)=1$.

\section{Proof.}

Since

$$
f_{p}(z)=\sum_{n=1}^{\infty} p a(n) e(p n z)=\sum_{n: p \mid n} p a(n / p) e(n z)
$$

we obtain

$$
\begin{aligned}
L_{f_{p} \otimes \eta_{j}}(s) & =p^{-s+1} \sum_{n=1}^{\infty} \frac{a(n) \overline{b_{j}(p n)}}{n^{s}} \\
& =p^{-s}\left(\lambda_{j}(p) L_{f \otimes \eta_{j}}(s)-p^{-s} \sum_{n=1}^{\infty} \frac{a(p n) \overline{b_{j}(n)}}{n^{s}}\right),
\end{aligned}
$$

using the relation (9) and the fact that $\lambda_{j}(p)$ is always real. Next, with relation (7),

$$
\begin{aligned}
\sum_{n=1}^{\infty} \frac{a(p n) \overline{b_{j}(n)}}{n^{s}} & =a(p) L_{f \otimes \eta_{j}}(s)-p \sum_{n: p \mid n} \frac{a(n / p) \overline{b_{j}(n)}}{n^{s}} \\
& =a(p) L_{f \otimes \eta_{j}}(s)-p^{-s+1} \sum_{n=1}^{\infty} \frac{a(n) \overline{b_{j}(p n)}}{n^{s}} \\
& =a(p) L_{f \otimes \eta_{j}}(s)-L_{f_{p} \otimes \eta_{j}}(s) .
\end{aligned}
$$

Hence

$$
\begin{aligned}
L_{f_{p} \otimes \eta_{j}}(s) & =p^{-s}\left(\lambda_{j}(p) L_{f \otimes \eta_{j}}(s)-p^{-s}\left(a(p) L_{f \otimes \eta_{j}}(s)-L_{f_{p} \otimes \eta_{j}}(s)\right)\right) \\
& =p^{-s} \lambda_{j}(p) L_{f \otimes \eta_{j}}(s)-p^{-2 s} a(p) L_{f \otimes \eta_{j}}(s)+p^{-2 s} L_{f_{p} \otimes \eta_{j}}(s) \\
& 13
\end{aligned}
$$


completing the proof.

So, the relation between the residues of $T_{p} Q^{*}$ and $Q^{*}$ we deduced from Proposition 9 reflects, in reality, a more global feature of the associated objects. On the other hand, the effect of the operators $U_{p}$ with $p \mid N$ on our series yields a more interesting relation among the convolution $L$-functions.

\section{Applying the Hecke operators $U_{p}$.}

We have been working with the Hecke operators $T_{p}$ for $p$ prime and not dividing the level $N$. We restrict our attention for a moment to the $\lambda_{j}$-eigenspace of $\Delta$ contained in $\mathcal{C}\left(\Gamma_{0}(N) \backslash \mathfrak{H}\right)$. This space can be decomposed into the space of newforms and the space of oldforms (cf. [AL],[Iw]). If $\eta(z)$ is a newform then as well as being an eigenfunction of the operators $T_{p}$ for $p \nmid N$ it is also automatically an eigenfunction of each $U_{p}$ for $p \mid N$. For our orthonormal family each $\eta_{j}$ may be chosen to be a newform or an oldform but we shall not need this assumption.

The following is the analogue of Lemma 8 of the previous section.

Lemma 11. Let $\Delta_{p}^{\prime}=\Delta_{p}-\left\{\left(\begin{array}{cc}p & 0 \\ 0 & 1\end{array}\right)\right\}$. For each prime $p$ with $p^{2} \mid N$ we have the equality

$$
\bigcup_{\substack{\rho \in \Delta_{p}^{\prime} \\ \tau \in H_{N}}} \Gamma_{\infty} \tau \rho=\bigcup_{\substack{\rho \in \Delta_{p}^{\prime} \\ \gamma \in H_{N / p}}} \Gamma_{\infty} \rho \gamma
$$

where the cosets on the left and right are disjoint. If the prime $p$ divides $N$ and $p^{2} \nmid N$ then we have

$$
\bigcup_{\substack{\rho \in \Delta_{p}^{\prime} \\ \tau \in H_{N}}} \Gamma_{\infty} \tau \rho \cup \bigcup_{\tau \in H_{N}} \Gamma_{\infty} \tau w_{p}=\bigcup_{\substack{\rho \in \Delta_{p}^{\prime} \\ \gamma \in H_{N / p}}} \Gamma_{\infty} \rho \gamma
$$

where again the unions are disjoint and $w_{p}$ is any matrix with determinant $p$ and of the form $\left(\begin{array}{ll}p a & 1 \\ N c & p\end{array}\right)$.

\section{Proof.}

We shall use Lemma 5 from [AL]: suppose the prime $p$ divides $N$ then let

$$
\Gamma_{0}(N / p, p)=\left\{\left(\begin{array}{ll}
a & b \\
c & d
\end{array}\right) \in \Gamma_{0}(N / p): p \mid b\right\}
$$

If $p \mid \frac{N}{p}$ then the matrices $S^{j}$ with $0 \leq j \leq p-1$ form a complete set of (disjoint) right coset representatives for $\Gamma_{0}(N / p, p)$ in $\Gamma_{0}(N / p)$. Further, if $p \nmid N / p$ we need the extra coset representative $\left(\begin{array}{cc}a p & 1 \\ c N / p & 1\end{array}\right)$ where $a$ and $c$ are integers satisfying the 
relation $a p-c N / p=1$. Denote this set of coset representatives $\Phi_{p}$. Thus

$$
\begin{gathered}
\left(\begin{array}{ll}
1 & 0 \\
0 & p
\end{array}\right) \Gamma_{0}(N / p)=\left(\begin{array}{ll}
1 & 0 \\
0 & p
\end{array}\right) \bigcup_{\alpha \in \Phi_{p}} \Gamma_{0}(N / p, p) \alpha= \\
\left(\begin{array}{ll}
1 & 0 \\
0 & p
\end{array}\right) \bigcup_{\alpha \in \Phi_{p}}\left(\begin{array}{ll}
1 & 0 \\
0 & p
\end{array}\right)^{-1} \Gamma_{0}(N)\left(\begin{array}{ll}
1 & 0 \\
0 & p
\end{array}\right) \alpha \\
=\bigcup_{\alpha \in \Phi_{p}} \Gamma_{0}(N)\left(\begin{array}{ll}
1 & 0 \\
0 & p
\end{array}\right) \alpha=\bigcup_{\rho \in \Delta_{p}^{\prime}} \Gamma_{0}(N) \rho \cup \Gamma_{0}(N) w_{p}
\end{gathered}
$$

where the term containing $w_{p}$ is included in the last line only if $p^{2} \nmid N$.

Now

$$
\left(\begin{array}{ll}
1 & 0 \\
0 & p
\end{array}\right) \Gamma_{0}(N / p)=\left(\begin{array}{ll}
1 & 0 \\
0 & p
\end{array}\right)\left(\begin{array}{ll}
1 & j \\
0 & 1
\end{array}\right) \Gamma_{0}(N / p)=\left(\begin{array}{ll}
1 & j \\
0 & p
\end{array}\right) \Gamma_{0}(N / p) .
$$

Hence,

$$
\bigcup_{\rho \in \Delta_{p}^{\prime}} \rho \Gamma_{0}(N / p)=\bigcup_{\substack{\rho \in \Delta_{p}^{\prime} \\ \gamma \in H_{N / p}}} \rho \Gamma_{\infty} \gamma=\bigcup_{\substack{\rho \in \Delta_{p}^{\prime} \\ \gamma \in H_{N / p}}} \Gamma_{\infty} \rho \gamma
$$

where the cosets in the last union are distinct but the cosets on the first one have multiplicity $p$. Therefore

$$
\left(\begin{array}{ll}
1 & 0 \\
0 & p
\end{array}\right) \Gamma_{0}(N / p)=\bigcup_{\substack{\rho \in \Delta_{p}^{\prime} \\
\gamma \in H_{N / p}}} \Gamma_{\infty} \rho \gamma
$$

and we have completed the proof.

To keep track of the level we will write $Q_{M}^{*}(z, s)=\sum_{\tau \in \Gamma_{\infty} \backslash \Gamma_{0}(M)} F(\tau z) \operatorname{Im}(\tau z)^{s}$.

Proposition 12. For each prime $p$ dividing $N$,

$$
U_{p} Q_{N}^{*}(z, s)=a(p) p^{-s} Q_{N / p}^{*}(z, s)-Q_{N}^{*}\left(w_{p} z, s\right)
$$

where the term containing $w_{p}$ is included only if $p^{2} \nmid N$.

\section{Proof.}

By definition

$$
\begin{aligned}
U_{p} Q_{N}^{*}(z, s) & =\sum_{\rho \in \Delta_{p}^{\prime}} \sum_{\tau \in H_{N}} F(\tau \rho z) \operatorname{Im}(\tau \rho z)^{s} \\
& =\sum_{\rho \in \Delta_{p}^{\prime}} \sum_{\gamma \in H_{N / p}} F(\rho \gamma z) \operatorname{Im}(\rho \gamma z)^{s}-\sum_{\tau \in H_{N}} F\left(\tau w_{p} z\right) \operatorname{Im}\left(\tau w_{p} z\right)^{s} \\
& =\sum_{\gamma \in H_{N / p}} \frac{1}{p^{s}} \operatorname{Im}(\gamma z)^{s} \sum_{\rho \in \Delta_{p}^{\prime}} F(\rho \gamma z)-Q_{N}^{*}\left(w_{p} z, s\right) \\
& =\sum_{\gamma \in H_{N / p}} \frac{1}{p^{s}} \operatorname{Im}(\gamma z)^{s} a(p) F(\gamma z)-Q_{N}^{*}\left(w_{p} z, s\right) \\
& =a(p) p^{-s} Q_{N / p}^{*}(z, s)-Q_{N}^{*}\left(w_{p} z, s\right) \\
& 15
\end{aligned}
$$


as in the statement of the proposition.

Using relation (11) as in the previous section we obtain the following. Let $V_{\lambda}^{M} \subset$ $\mathcal{C}\left(\Gamma_{0}(M) \backslash \mathfrak{H}\right)$ denote the $\lambda$-eigenspace of the Laplacian $\Delta$ and set $B_{\lambda}^{M}$ to be any orthonormal basis (w.r.t $\langle,\rangle_{M}$ ), of this space. Then we have

Proposition 13. Suppose the prime $p$ divides $N$. Set $W_{p} \phi(z):=\phi\left(w_{p} z\right)$ and define $\tilde{U}_{p}$ to be $U_{p}$ if $p^{2} \mid N$ or $U_{p}+W_{p}$ if $p^{2} \nmid N$. Then

$$
\sum_{\phi \in B_{\lambda}^{N}} L_{f \otimes \phi}\left(s_{\lambda}\right) \tilde{U}_{p} \phi(z)=\frac{a(p)}{p^{s_{\lambda}}} \sum_{\psi \in B_{\lambda}^{N / p}} L_{f \otimes \psi}\left(s_{\lambda}\right) \psi(z)
$$

where $\lambda=s_{\lambda}\left(1-s_{\lambda}\right)$.

This formula may be verified in simple cases and, as before, it seems to be true for all $s \in \mathbb{C}$ not just $s=s_{\lambda}$. Proving this seems difficult though. In another direction it should also be possible to prove a completely holomorphic version of this proposition with $B_{\lambda}^{M}$ replaced by an orthonormal basis for the holomorphic cusp forms $S_{k}(M)$.

We conclude this section by mentioning an application of our last Proposition. Suppose that $\phi(z) \in \mathcal{C}\left(\Gamma_{0}(1) \backslash \mathfrak{H}\right)$ is a Maass cusp form with $\Delta \phi=\lambda \phi$. Let $W^{p^{k}}$ be the vector space generated by $\phi(z)$ and its 'descendants', $\phi_{p^{i}}(z):=\phi\left(p^{i} z\right)$ for $i=1,2, \ldots, k$. Note that $\phi_{p^{i}} \in \mathcal{C}\left(\Gamma_{0}\left(p^{i}\right) \backslash \mathfrak{H}\right)$ and $\Delta \phi_{p^{i}}=\lambda \phi_{p^{i}}$.

To make the presentation easier we re-normalize our inner product and define

$$
\left\langle\xi_{1}, \xi_{2}\right\rangle=\left[\Gamma_{0}(1): \Gamma_{0}(M)\right]^{-1}\left\langle\xi_{1}, \xi_{2}\right\rangle_{M},
$$

where it is understood that if $\xi_{i} \in \mathcal{C}\left(\Gamma_{0}\left(l_{i}\right) \backslash \mathfrak{H}\right)$ for $i=1,2$ then $l_{i} \mid M$. Let us assume that $\langle\phi, \phi\rangle=1$ and that $T_{p} \phi=\lambda_{p} \phi$. It should be noted that the Maass forms $\phi_{p^{i}}$ do not form an orthonormal basis for $W^{p^{k}}$, for example it may be shown that $\left\langle\phi, \phi_{p}\right\rangle=\lambda_{p} /(p+1)$.

We construct an orthonormal basis $\left\{\psi_{0}, \psi_{1}, \ldots, \psi_{k}\right\}$ for $W^{p^{k}}$ using the GramSchmidt process where at each stage $\left\{\psi_{0}, \psi_{1}, \ldots, \psi_{i}\right\}$ is a basis for $W^{p^{i}}$. Now Proposition 13 implies that

$$
L_{f \otimes \psi_{i}}\left(s_{\lambda}\right) U_{p} \psi_{i}(z)=\frac{a(p)}{p^{s_{\lambda}}} L_{f \otimes \psi_{i-1}}\left(s_{\lambda}\right) \psi_{i-1}(z)
$$

for $i \geq 3$. Combining this with the fact that $U_{p} \phi_{p^{i}}=p \phi_{p^{i-1}}$ for $i \geq 1$ and a few other results we deduce

Theorem 14. Let $\alpha=\left((p+1)^{2}-\lambda_{p}^{2}\right)^{-1 / 2}$ and $\beta=(p+1)^{1 / 2}(p-1)^{-1 / 2} \alpha$ where $\alpha, \beta \in \mathbb{R}$, then

$$
\begin{aligned}
\psi_{0} & =\phi \\
\psi_{1} & =\alpha\left(-\lambda_{p} \phi+(p+1) \phi_{p}\right) \\
\text { and } \quad \psi_{i} & =\beta\left(\phi_{p^{i-2}}-\lambda_{p} \phi_{p^{i-1}}+p \phi_{p^{i}}\right)
\end{aligned}
$$


for $i \geq 2$, gives an orthonormal basis $\left\{\psi_{0}, \psi_{1}, \ldots, \psi_{k}\right\}$ for the space spanned by $\phi, \phi_{p}, \ldots, \phi_{p^{k}}$ where $\phi$ is a Maass cusp form for the full modular group and $\phi_{p^{i}}(z)=$ $\phi\left(p^{i} z\right)$.

We may also write $W^{p^{k}}=W_{\text {old }} \oplus W_{\text {new }}$. The space of oldforms, $W_{\text {old }}$, is spanned by $\phi_{p^{i}}$ with $1 \leq i \leq k$ and $W_{\text {new }}$, the space of newforms, is the orthogonal complement of $W_{\text {old }}$. We see that $\phi_{i} \in W_{\text {old }}$ for $i \geq 3$. If we rearrange terms and set

$$
\begin{aligned}
\xi_{0} & =\beta\left(p \phi-\lambda_{p} \phi_{p}+\phi_{p^{2}}\right), \\
\xi_{1} & =\phi_{p} \\
\text { and } \quad \xi_{2} & =\alpha\left(-\lambda_{p} \phi_{p}+(p+1) \phi_{p^{2}}\right)
\end{aligned}
$$

then it may be shown that $\left\{\xi_{0}\right\}$ and $\left\{\xi_{1}, \xi_{2}, \psi_{3}, \ldots, \psi_{k}\right\}$ are orthonormal bases for $W_{\text {new }}$ and $W_{\text {old }}$ respectively when $k \geq 2$.

Only brief sketches of the proofs have been given above. The second author hopes to return to these topics in a future work.

\section{Other Hecke operators.}

An alternative way to define the space of functions $G^{*}$ (and the one appearing in [G1]) is to fix $f \in S_{k}(M)$ and $G \in M_{m}(N, \chi)$ and to consider the vector space $M(f, G)$ of functions $G^{*}: \mathfrak{H} \rightarrow \mathbb{C}$ of polynomial growth at the cusps such that for some $c \in \mathbb{C}$ we have $\left.G^{*}\right|_{m-(k-2)}(\gamma-1)=c \cdot r_{f}(\gamma) \cdot G$ for all $\gamma \in \Gamma_{0}(N)$.

If we fix only $f \in S_{k}(M)$, we can set $M(f)=\left\{G^{*}: \mathfrak{H} \rightarrow \mathbb{C}\right.$ of polynomial growth at the cusps such that for some $G \in M_{m}(N, \chi)$ we have: $\left.G^{*}\right|_{m-(k-2)}(\gamma-1)=r_{f}(\gamma) \cdot G$ for all $\left.\gamma \in \Gamma_{0}(N)\right\}$. In an analogous manner, we can define $M(G)$ for a fixed $G \in$ $M_{m}(N, \chi)$.

It is possible to define Hecke operators on $M(f, G), M(f), M(G)$ which are compatible with each other. To this end, we prove first the following

Proposition 15. For non-zero $f \in S_{k}(M), G \in M_{m}(N)$ we have:

$$
\begin{aligned}
& M(f) \cong M_{m}(N) \oplus M_{m-(k-2)}(N), \\
& M(G) \cong S_{k}(M) \oplus M_{m-(k-2)}(N) \text { and } \\
& M(f, G) \cong \mathbb{C} \oplus M_{m-(k-2)}(N) .
\end{aligned}
$$

\section{Proof.}

Suppose that $\left.G^{*}\right|_{m-(k-2)}(\gamma-1)=r_{f}(\gamma) G$ for some $G \in M_{m}(N)$.Then, if $\tilde{f}(z)$ denotes the Eichler integral $\int_{i \infty}^{z} f(w)(w-z)^{k-2} d w$, there is a $g \in M_{m-(k-2)}(N)$ such that $G^{*}=\tilde{f} \cdot G+g$ because $\tilde{f} \cdot G$ satisfies the above equation too, so $G^{*}-\tilde{f} \cdot G \in$ $M_{m-(k-2)}(N)$. This expression is unique because if $G^{*}=\tilde{f}(z) G^{\prime}(z)+g^{\prime}(z)$ for some other $G^{\prime}, g^{\prime}$ then, $\tilde{f}\left(G^{\prime}-G\right)=g-g^{\prime}$ and hence

$$
\left.\left(\tilde{f}\left(G^{\prime}-G\right)\right)\right|_{m-(k-2)}(\gamma-1)=\left.\left(g-g^{\prime}\right)\right|_{m-(k-2)}(\gamma-1)
$$


for all $\gamma \in \Gamma_{0}(N)$. The equality $\left.\tilde{f}\right|_{m-(k-2)}(\gamma-1)=r_{f}(\gamma)$ then implies that for all $z \in \mathfrak{H}, \gamma \in \Gamma_{0}(N)$, we have $r_{f}(\gamma)(z)\left(G(z)-G^{\prime}(z)\right)=0$. Now choose a $z_{0} \in \mathfrak{H}$ such that $G\left(z_{0}\right)-G^{\prime}\left(z_{0}\right) \neq 0$ and a $\gamma_{0}$ such that $r_{f}\left(\gamma_{0}\right) \neq 0$. (The existence of such a $\gamma_{0}$ is guaranteed by the Eichler-Shimura isomorphism.) Then $G-G^{\prime}$ does not vanish at $S^{l} z_{0}(l=0, \ldots, k-2)$ either, therefore $r_{f}\left(\gamma_{0}\right)$ must vanish there, which is impossible since $r_{f}\left(\gamma_{0}\right)$ is a polynomial of degree at most $k-2$.

Similarly, each element $G^{*}$ of $M(G)$ can be written uniquely in the form $\tilde{f}(z) G(z)+$ $g(z)$ for some $f \in S_{k}(M)$ and $g \in M_{m-(k-2)}(N)$ and each element $G^{*}$ of $M(f, G)$ can be written uniquely in the form $c \tilde{f}(z) G(z)+g(z)$ for some $c \in \mathbb{C}$ and $g \in$ $M_{m-(k-2)}(N)$.

In view of this proposition, for any $f \in S_{k}(M)$ and any $p$ with $p \nmid N$ we can define a Hecke operator $T_{p}(f): M(f) \rightarrow M(f)$ by the formula

$$
T_{p}(f) G^{*}=\tilde{f} \cdot T_{p} G+T_{p} g
$$

for $G^{*}=\tilde{f} G+g$. By $T_{p}$ on the RHS we denote the usual Hecke operator on $M_{m}(N)$ or $M_{m-(k-2)}(N)$ (according to which functions it is applied on). Obviously, $T_{p}(f) G^{*}$ belongs to $M(f)$ satisfying the equation:

$$
\left.\left(T_{p}(f) G^{*}\right)\right|_{m-(k-2)}(\gamma-1)=r_{f}(\gamma) T_{p} G .
$$

This map induces a graded morphism of degree 0 from $\oplus_{f \in S_{2}(M)} M(f)$ to itself.

Similarly, for each $G \in M_{m}(N)$, we can define a Hecke operator $T_{p}(G): M(G) \rightarrow$ $M(G)$ by the formula

$$
T_{p}(G) G^{*}=\left(\tilde{T_{p}} f\right) \cdot G+T_{p} g
$$

for any $p$ with $(p, N)=1$ and with the analogous convention on the notation of the usual Hecke operators as above. Thus $T_{p}(G) G^{*} \in M(G)$ and it satisfies the equation: $\left.\left(T_{p}(G) G^{*}\right)\right|_{m-(k-2)}(\gamma-1)=r_{T_{p} f}(\gamma) G$. This map induces a graded morphism of degree 0 from $\oplus_{G \in M_{k}(N)} M(G)$ to itself.

Finally, we can set

$$
T_{p}(f, G) G^{*}=c \tilde{f} G+T_{p} g
$$

for $G^{*}(z)=c \tilde{f}(z) G(z)+g(z) \in M(f, G)$ and it is easy to see that $T_{p}(f, G) G^{*}$ will belong to $M(f, g)$ satisfying the same transformation law as $G^{*}$. Again, this map induces a graded morphism of degree 0 from $\oplus_{G \in M_{k}(N)} M(f, G)$ to itself.

It can be seen directly from their definitions that the maps induced by $T_{p}(f)$, $T_{p}(G)$ and $T_{p}(f, G)$ commute with each other. The spaces we construct this way:

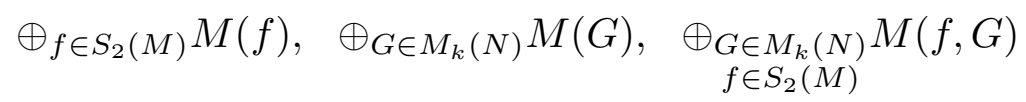

are isomorphic (but via isomorphisms which do not respect the grading of course).

As an example, the Eisenstein series $E^{*}(z, s)$ of Section 3 is an element of $M(f, E)$. It may be expressed as

$$
E^{*}(z, s)=Q^{*}(z, s)-\tilde{f}(z) E(z, s)
$$


with $Q^{*}(z, s)=\sum_{\tau \in \Gamma_{\infty} \backslash \Gamma} F(\tau z) \operatorname{Im}(\tau z)^{s}$ as before. So we have

$$
T_{p}(f, E) E^{*}(z, s)=T_{p} Q^{*}(z, s)-\tilde{f}(z) E(z, s) .
$$

Therefore an immediate corollary of Proposition 9 is

Corollary 16. For $p$ not dividing $N$,

$$
\begin{aligned}
T_{p}(f, E) E^{*}(z, s) & =a(p) p^{-s}\left(E^{*}(z, s)-F(z) E(z, s)\right) \\
& +\left(p^{s}-p^{-s}\right)\left(E^{*}\left(z, s ; f_{p}\right)-F(p z) E(z, s)\right)+F(z) E(z, s) .
\end{aligned}
$$

By comparison $T_{p} E(z, s)=\left(p^{1-s}+p^{s}\right) E(z, s)$.

\section{REFERENCES}

[A] Ash, A., Parabolic cohomology of arithmetic subgroups of $S L(2, \mathbb{Z})$ with coefficients in the field of rational functions on the Riemann sphere., Amer. J. Math. 111 (1989), 35-51.

[AL] Atkin, A., Lehner, J., Hecke operators on $\Gamma_{0}(m)$, Math. Ann. 185 (1970), 134-160.

[CZ] Choie, Yj., Zagier, Rational period functions for PSL(2, Z), Contemp. Math. 143 (1993), 89-108.

[D1] Diamantis, N., Values of higher derivatives of L-functions, Forum Math. 11 (1999), 229-252.

[D2] - Hecke operators and derivatives of L-functions, (to appear).

[DI] Deshouillers, J.M., Iwaniec, H., Rankin-Selberg zeta functions, Contemp. Math. 53 (1986), 51-95.

[G1] Goldfeld, D., Zeta functions formed with modular symbols, (to appear).

[G2] - The distribution of modular symbols, Number Theory in Progress (Proceedings of the International Conference organized by the S. Banach Intern. Math. Center in honor of Schinzel in Zakopane, Poland, June 30-July 9, 1997) (1999).

[G3] - Special values of derivatives of L-functions, CMS Conf. Proc. 15 (1995), 245-282.

[G4] Modular elliptic curves and diophantine problems, Proc. of the First Canadian Number Theory Assoc., Banff, Canada (1990), 157-175.

[Iw] Iwaniec, H., Introduction to the spectral theory of automorphic forms, Bibl. Rev. Mat. Iber., Madrid (1995).

[K] Knopp, M., Rational period functions of the modular group, Duke. Math. J. 45 (1978), 47-62.

[L] Luo, W., On the non-vanishing of Rankin-Selberg L-functions, Duke Math J. 69 (1993), 411-427.

[M] Manin, Y. T., Periods of parabolic points and p-adic Hecke series, Math. Sb. (1973), 371-393.

[O'S1] O'Sullivan, C., Properties of Eisenstein series formed with modular symbols, Ph.D Thesis, Columbia University (1998).

[O'S2] _ Properties of Eisenstein series formed with modular symbols, Crelle's Journal (1999).

[PS] Phillips, R.S., Sarnak, P., On cusp forms for cofinite subgroups of PSL(2,R), Invent. Math. 80 (1985), 339-364.

[S] Shimura, G., Introduction to the Arithmetic Theory of Automorphic Functions, Princeton University Press (1971). 